\title{
Screening for Resistance to Verticillium Wilt in Spinach and Isolation of Verticillium dahliae from Seed of Spinach Accessions
}

\author{
Maria I. Villarroel-Zeballos ${ }^{1}$, Chunda Feng, and Angela Iglesias \\ Department of Plant Pathology, University of Arkansas, Fayetteville, \\ AR 72701
}

\author{
Lindsey J. du Toit \\ Department of Plant Pathology, Washington State University Mount Vernon \\ NWREC, Mount Vernon, WA 98273
}

\author{
James C. Correll \\ Department of Plant Pathology, University of Arkansas, Fayetteville, \\ AR 72701
}

Additional index words. germplasm, Spinacia oleracea, Spinacia turkestanica

\begin{abstract}
Verticillium dahliae is a pathogen of spinach (Spinacia oleracea) during spinach seed crop production but not in vegetative leafy spinach crops, because plants remain asymptomatic until bolting has been initiated (conversion from vegetative to reproductive growth). The objectives of this research were to evaluate a set of USDA spinach germplasm accessions for resistance to Verticillium wilt and to determine the range in incidence of natural seed infection/infestation in a subset of the USDA spinach accessions screened for resistance. A total of 120 Spinacia spp. accessions from the USDA North Central Regional Plant Introduction Station spinach germplasm collection and 10 commercial $S$. oleracea hybrids were screened for resistance to $V$. dahliae in Trials 1 , 2, and 3 in 2006, 2007, and 2008, respectively, in greenhouse evaluations. Each accession was grown in either $V$. dahliae-infested or non-infested potting mix and rated weekly on a seven-step scale from 1 week before bolting to 4 weeks after bolting. A wide range of disease severity ratings was observed among the accessions evaluated. Most of the accessions were highly susceptible. There was no evidence of qualitative resistance but some showed greater levels of quantitative resistance than others. Plants in soil infested with $\boldsymbol{V}$. dahliae senesced faster and had significantly reduced biomass compared with plants in non-infested soil of the same accession. In addition, in Trial 2 (2007), 34\% (20 of 59) of the seed samples assayed of the accessions were infested or infected with $\mathrm{V}$. dahliae; and in Trial 3, (2008) 16\% (21 of 130) of the seed samples of the USDA accessions evaluated were infested or infected with $V$. dahliae, $V$. tricorpus, or Gibellulopsis nigrescens (formerly known as $V$. nigrescens). These results are valuable for characterizing potential genetic variability within spinach germplasm for resistance to $\mathrm{V}$. dahliae.
\end{abstract}

Spinach (Spinacia oleracea L.) is an economically valuable crop grown worldwide (Morelock and Correll, 2008) and is affected by a number of biotic and abiotic disorders that can impact yield and quality (Correll

\footnotetext{
Received for publication 7 Feb. 2012. Accepted for publication 26 June 2012

This research was supported in part by the California Spinach Research Committee, the California Leafy Greens Research Board, and the American Seed Trade Association through the American Seed Research Foundation grant entitled "Management of Verticillium in Spinach."

We thank the USDA-NCRPIS for supplying the spinach germplasm accessions and Chriseed, Pop Vriend, Rijk Zwaan, and Seminis seed companies for providing commercial hybrids for our research. ${ }^{1}$ Present Address: Department of Plant Pathology, University of Nebraska-Lincoln, Lincoln, NE 68588. ${ }^{2}$ To whom reprint requests should be addressed; e-mail jcorrell@uark.edu.
}

et al., 1994). Several soilborne pathogens affect the production of spinach leaf and seed crops (Correll et al., 1994). Although Verticillium dahliae causes Verticillium wilt of more than 200 species of vegetables, field crops, and trees, the fungus was not, until recently, recognized as a pathogen of spinach (du Toit et al., 2005). However, V. dahliae was recovered from spinach seed from New York in 1962 (Snyder and Wilhelm, 1962), and seed transmission of $V$. dahliae was reported in The Netherlands (van der Spek, 1972, 1973) and Canada (Sackston and Sedun, 1982). Verticillium wilt is not recognized as a disease in the commercial fresh market or processing spinach crops in the United States or elsewhere (Correll et al., 1994). However, $V$. dahliae was recently recovered from spinach plants grown for seed production and demonstrated to be pathogenic on spinach (du Toit et al., 2005). Interestingly, Verticillium wilt symptoms did not appear on inoculated plants until after the reproductive phase of the plant life cycle had been initiated (bolted) by increasing the duration of daylength (du Toit et al., 2005). Symptoms of Verticillium wilt of spinach include leaf chlorosis, sometimes interveinal chlorosis, and wilting, starting with the lower (older) leaves and moving up the plant, stunting, a very light brown vascular discoloration of the roots and crown, and accelerated plant death (du Toit et al., 2005; Koike et al., 2007).

Spinach seed production is limited to areas where environmental conditions include long summer days to induce flowering and moderate summer temperatures (Metzger and Zeevaart, 1985). Denmark produces $\approx 75 \%$ of the world's spinach seed supply followed by the Pacific Northwest region of the United States (20\%) (Correll et al., 2011; Daehnfeldt, 2007; Foss and Jones, 2005). In the Pacific Northwest, $\approx 600$ to 1600 ha of spinach seed crops are grown annually, representing $25 \%$ to $50 \%$ of the U.S. spinach seed supply (Correll et al., 2011; Daehnfeldt, 2007). V. dahliae was recovered from $89 \%$ of 75 spinach seed lots produced in Washington, The Netherlands, and Denmark (du Toit et al., 2005) as well as seed lots produced in Chile and New Zealand (du Toit, unpublished data). Seed lots were found with incidences of $V$. dahliae infection up to $84.8 \%$ per lot, and seed-to-seed transmission was demonstrated by planting infected seed lots and taking the plants that grew out to flowering and seed set (du Toit et al., 2005). Although symptoms of Verticillium wilt have not been observed in fresh market and processing spinach crops, the seedborne nature of the pathogen is a significant concern because infested or infected spinach seed could potentially introduce $V$. dahliae into production fields and result in disease problems in subsequent crops (e.g., spinach and potato crops grown in rotation in the Pacific Northwest) (Atallah et al., 2010), or planting a spinach seed crop into soil infested from a previous crop could potentially lead to infection of the harvested seed (du Toit et al., 2005).

Verticillium wilt is particularly difficult to manage because microsclerotia of the pathogen can survive in the soil for long periods of time, and even a few microsclerotia per gram of soil can result in significant disease incidence in some crops under favorable environmental conditions (Grogan et al., 1979; Nicot and Rouse, 1987). Although a wide range of management options are available to help control Verticillium wilt, perhaps the most effective and economical practice to control $V$. dahliae is the use of plant resistance (Atibalentja and Eastburn, 1998; Diwan et al., 1999; Harrison and Beckman, 1982; Vallad and Subbarao, 2008; Wilhelm, 1955). Resistance to $V$. dahliae has been identified in various crops, including tomato (Diwan et al., 1999), sunflower (Klisiewicz, 1981), cotton (Harrison and Beckman, 1982), cacao (Resende et al., 1996), artichoke (Cirulli et al., 1994), horseradish (Atibalentja and Eastburn, 1998), eggplant (Bejarano-Alcazar et al., 1999), lettuce (Hayes et al., 2011), and potato (Jansky 
et al., 2004). Both single dominant gene and quantitative resistance have been identified in numerous crop hosts (Hayes et al., 2011; Schaible et al., 1951; Simko et al., 2004). Screening the USDA spinach germplasm collection has been valuable for identifying resistance to several diseases including downy mildew (Brandenberger et al., 1991), Fusarium wilt (Laguna, 2000), and leaf spot (Mou et al., 2008). However, there have been no published studies on resistance screening to Verticillium wilt in spinach to date. Therefore, the objectives of this study were to: 1) quantify the reactions of a subset of the USDA spinach germplasm collection to inoculation with $V$. dahliae; and 2) evaluate the incidence of natural infestation/infection by $V$. dahliae and other Verticillium species on spinach seed lots of the USDA germplasm collection. Preliminary results have been presented (VillarroelZeballos et al., 2006, 2007, 2008).

\section{Materials and Methods}

\section{Screening for resistance to $V$. dahliae}

Plant material and plant production. A total of 120 accessions of Spinacia spp. from the U.S. Department of Agriculture-North Central Regional Plant Introduction Station (USDA-NCRPIS) spinach germplasm collection, selected from a total of 360 accessions to represent diverse geographical origins, were screened for resistance to $V$. dahliae in 2006 (Trial 1), 2007 (Trial 2), and 2008 (Trial 3). Additionally, 10 commercial spinach hybrids commonly used as differentials for race identification of strains of the downy mildew pathogen, Peronospora farinosa f. sp. spinaceae, were included in the 2007 and 2008 resistance screening trials. Seed of each spinach genotype were planted and incubated in a greenhouse with 12 -h daylength at $25^{\circ} \mathrm{C}$ average temperature. The plants were watered daily and fertilized with Peters $20 \mathrm{~N}-20 \mathrm{P}-20 \mathrm{~K}$ every 8 to $10 \mathrm{~d}$ at a rate of $\approx 2 \mathrm{~g} \cdot \mathrm{L}^{-1}$. The tests were planted on 17 Apr. 2006, 14 Mar. 2007, and 28 Jan. 2008 for Trials 1, 2, and 3, respectively. Spinach is a long daylength plant whereby flowering is initiated as daylength increases (Metzger and Zeevaart, 1985). Although there is a lot of genetic variability in the number of hours required to induce flowering in spinach, the 2006 and 2007 experiments were planted in early spring to allow the natural increase in daylength that time of year to induce bolting $\approx 2$ to 6 weeks after planting. In 2008, seeds were planted during the winter to let the plants grow for $30 \mathrm{~d}$ and then induced to bolt by increasing the photoperiod in the greenhouse to $20 \mathrm{~h} \cdot \mathrm{d}^{-1}$ for $8 \mathrm{~d}$ using supplemental lighting. This resulted in all of the genotypes bolting at a similar number of days after planting to avoid confounding daylength sensitivity of the genotypes with the timing of symptom development.

Isolate and inoculum production. An isolate of $V$. dahliae (VD1), demonstrated to be a pathogen of spinach (du Toit et al., 2005) and belonging to VCG 2B (Iglesias-Garcia, 2011), was used to infest soil for the resistance screening test. A $2-\mathrm{mm}^{2}$ piece of colonized filter paper on which isolate VD1 was stored was plated on potato dextrose agar (PDA). After 2 weeks, colonies were transferred to new plates of PDA and incubated for $12 \mathrm{~d}$. Erlenmeyer flasks ( $1 \mathrm{~L})$ containing sterile vermiculite amended with minimal medium nutrient solution (Korolev and Katan, 1997) were each inoculated with 10 mycelial plugs taken from the actively growing edge of a colony of VD1. Non-infested, sterile vermiculite amended with minimal medium nutrient solution was prepared for the non-inoculated control treatment of each spinach accession. The inoculated vermiculite was incubated in the dark at room temperature $\left(22\right.$ to $\left.24{ }^{\circ} \mathrm{C}\right)$ for $30 \mathrm{~d}$, by which time microsclerotia of $V$. dahliae had developed in the vermiculite. Scott's RediEarth potting mix was steamed for 30 min at $71^{\circ} \mathrm{C}$ and then amended at a rate of 1.0 $\mathrm{g}$ vermiculite inoculum $/ \mathrm{kg}$ potting mix. This potting mix inoculum was mixed and watered periodically for 1 week before planting.

Experimental design and disease severity evaluations. The resistance screening experiment was set up as a split plot design with soil treatment (infested or non-infested) as the whole plot and 120 spinach accessions in 2006 or 130 lines in each of 2007 and 2008 as the subplots with six replications (pots)/ treatment combination and three plants/pot. Plants were observed every $7 \mathrm{~d}$ to determine the timing of onset of bolting and development of symptoms of Verticillium wilt. Plant gender (spinach is dioecious) was recorded at the same time as the disease ratings. Each plant was rated for disease severity 35 or $36 \mathrm{~d}$ after planting $(\approx 1$ week before bolting) and weekly thereafter for $\approx 3$ weeks after bolting $(35,43,51$, and $62 \mathrm{~d}$ after planting in Trial 1 ; $38,50,57$, and $64 \mathrm{~d}$ in Trial 2; and 36, 47, 51, and $70 \mathrm{~d}$ in Trial 3 ) using the disease severity scale described by du Toit et al. (2005) with modifications. Briefly, each plant was rated on a seven-step scale, where: $0=$ no symptoms ( $0 \%$ disease severity), $1=1 \%$ to $10 \%$ of the plant showing chlorosis and necrosis, $2=$ $11 \%$ to $25 \%$ chlorosis and necrosis, $3=26 \%$ to $50 \%$ chlorosis and necrosis, $4=51 \%$ to $75 \%$ chlorosis and necrosis, $5=76 \%$ to $99 \%$ chlorosis and necrosis, and $6=100 \%$ chlorosis and necrosis. The scoring was performed on each plant, and the results were converted to percentage values (midpoints). Midpoints of the severity ratings were then averaged per pot. The disease rating 50 to $51 \mathrm{~d}$ after planting was determined to be optimum for disease development, before potential confounding with natural senescence. Thus, analysis of variance (ANOVA) was performed for the disease rating done 50 to $51 \mathrm{~d}$ after planting using PROC MIXED (SAS Institute Inc., Cary, NC) to obtain LSMeans estimates for each entry. However, because there was very little variability in the replication means of the non-infested data, they were excluded from the final analysis, and an arithmetic average was obtained instead. As a result, the ANOVA was based on a randomized block design with random blocks and entry as the fixed factor. The spinach accessions were ranked for disease severity in all three trials and identified as highly susceptible or displaying some degree of resistance to $V$. dahliae. Accessions with less than $25 \%$ mean disease severity were considered partially resistant, whereas accessions with a mean disease severity greater than $75 \%$ were considered highly susceptible.

Re-isolation of the pathogen. All plants that grew in infested potting mix senesced after bolting was initiated. In addition, some plants in the non-infested potting mix senesced after bolting was triggered. Consequently, in 2006, isolations were made from plants in each of six replications of three spinach lines (three plants/replication) selected at random from both the infested and noninfested treatments. In 2007, isolations were completed for $50 \%$ of the plants of each genotype in the non-infested soil (nine plants/genotype) and from plants of 10 accessions selected randomly from the infested potting mix. The nine plants represented three replications (pots) of the most severely symptomatic plants of each genotype. Isolations were carried out using five petiole pieces/ plant. In 2008, isolations were conducted on all 130 accessions by selecting the most symptomatic plant of each accession in the noninfested treatment and from 10 accessions selected randomly from the infested potting mix treatment. The isolations were performed by surface-disinfecting each leaf petiole for 1 min with $0.5 \% \mathrm{NaOCl}$, rinsing the petiole with deionized water, and then plating petiole sections on water agar. Each petiole was then examined microscopically $(40 \times$ to $500 \times$ magnification) for development of conidiophores and/or microsclerotia of $V$. dahliae.

In the 2007 trial, aboveground biomass of the plants of each line from the infested and non-infested potting mix treatments was also measured. At $64 \mathrm{~d}$ after planting (16 May 2007), all plants were cut at the crown and the aboveground tissues dried in a forced-air oven at $60^{\circ} \mathrm{C}$. Dry biomass/plant was analyzed by ANOVA on SAS using PROC MIXED, and least square means were generated from the model. Additionally, the reduction in biomass of plants in infested soil vs. biomass of plants in non-infested soil was calculated/ plant as follows:

\section{[(Dry plant biomass in non - infested soil) \\ - (Dry plant biomass in infested soil)]/}

(Dry plant biomass in non - infested soil).

Pearson's correlation coefficient between aboveground biomass reduction and severity of Verticillium wilt was calculated using JMP software.

\section{Freeze-blotter seed health assay}

To assess the potential that seedborne inoculum may have contributed inoculum of Verticillium to infection of plants growing in non-infested potting mix, a freeze-blotter seed health assay for $V$. dahliae (du Toit et al., 2005) was performed on a small sample of seed from the original seed lots obtained from the USDA-NCRPIS for all accessions that had a disease severity rating greater than $0 \%$ 
when evaluated 50 to $51 \mathrm{~d}$ after planting in Trial 2. Approximately 10 to 20 seeds (depending on how much seed was available) from each of the 59 genotypes were examined in 2007. In 2008, 20 seeds/accession of each of the 130 accessions were evaluated for the presence/absence of $V$. dahliae. Seed from three different lots with known $V$. dahliae infection levels of $84 \%, 45 \%$, and $25 \%$ (du Toit et al., 2005) were included as control samples in the freeze-blotter seed health tests in 2007 and 2008.

Isolates recovered from seed were examined for colony morphology, colony pigmentation, conidiophore length and appearance, and microsclerotial production (Domsch et al., 1993; Smith, 1965). In addition, representative isolates of the different species putatively identified based on morphology were examined based on the internally transcribed spacer (ITS) region of ribosomal DNA (rDNA) (White et al., 1990). The region was amplified using the ITS primers ITS $1\left(5^{\prime}\right.$-TCCGTAGGTGA ACCTGCGG-3') and ITS4 (5' -TCCTCCGC
TTATTGATATGC-3'). The amplified product was subsequently sequenced and, existing sequences in Genbank of various species of Verticillium were examined for similarities to the ITS rDNA sequences.

\section{Results}

Screening for resistance to Verticillium wilt. Symptoms of Verticillium wilt were initially observed 4 to 5 weeks after planting, after the plants had started to bolt. Symptoms started on the oldest leaves as interveinal chlorosis and progressed as the plants aged. Severity of interveinal chlorosis increased and spread to most leaves of each inoculated plant, and many leaves became necrotic and died. A wide range in disease severity values was observed 51, 50, and $51 \mathrm{~d}$ after planting when the plants were rated for disease severity in Trials 1, 2, and 3, respectively. Verticillium wilt severity ranged from $5.7 \%$ to $91.1 \%$, $5.7 \%$ to $99.3 \%$, and $5.5 \%$ to $93.8 \%$ in Trials 1,2 , and 3 , respectively. No accession appeared immune in any of the trials (data not shown; for complete data set, see VillarroelZeballos, 2007). In the non-infested soil treatment, most of the accessions (104 of 120 in Trial 1, 109 of 130 in Trial 2, and 126 of 130 in Trial 3) had low disease severity ratings (less than 20\%) (data not shown).

ANOVAs for Trial 1 (2006) indicated statistically significant effects of spinach accession $(P<0.0001)$. Accessions with the lowest disease severity ratings were PI 193618, Ames 26243, PI 604789, PI 179591, PI 261789, PI 319220, and PI 286435 (Table 1). Seven accessions were rated as highly susceptible to $V$. dahliae (disease severity $75 \%$ or greater), including NSL 28217, PI 358251, PI 165560, PI 361127, PI 173972, NSL 101759, and PI 200882 (Table 1).

ANOVA for Trial 2 (2007) indicated statistically significant effects for spinach accession $(P<0.0001)$. Twenty-seven accessions were rated as resistant to Verticillium wilt (disease severity $25 \%$ or less). The lowest disease severity ratings were observed for PI

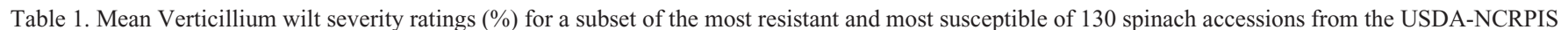
germplasm collection that were planted into potting medium infested or not infested with Verticillium dahliae in each of three greenhouse trials. ${ }^{\mathrm{z}}$

\begin{tabular}{|c|c|c|c|c|c|c|c|c|c|c|c|}
\hline \multirow{2}{*}{$\begin{array}{l}\text { Spinach } \\
\text { entry }^{y}\end{array}$} & \multirow{2}{*}{$\begin{array}{c}\text { USDA } \\
\text { accession no. }\end{array}$} & \multirow[b]{2}{*}{ Origin } & \multicolumn{3}{|c|}{ Trial 1 (2006) } & \multicolumn{3}{|c|}{ Trial 2 (2007) } & \multicolumn{3}{|c|}{ Trial 3 (2008) } \\
\hline & & & Infested $^{\mathrm{w}}$ & $\operatorname{Rank}^{\mathrm{u}}$ & Non-infested $^{t}$ & Infested $^{\mathrm{w}}$ & $\operatorname{Rank}^{\mathrm{u}}$ & Non-infested $^{t}$ & Infested $^{\mathrm{w}}$ & $\operatorname{Rank}^{\mathrm{u}}$ & Non-infested \\
\hline 45 & PI 175931 & Turkey & 21.9 & 30 & 5.0 & 5.7 & 1 & 0.0 & 8.3 & 6 & 0.3 \\
\hline 4 & Ames 26243 & China & 7.1 & 2 & 5.0 & 10.6 & 2 & 0.0 & 9.0 & 8 & 0.0 \\
\hline 25 & PI 163309 & India & 16.7 & 12 & 6.4 & 11.7 & 3 & 0.3 & 20.2 & 34 & 0.0 \\
\hline 75 & PI 261789 & France & 8.9 & 5 & 6.2 & 12.3 & 4 & 0.0 & 14.3 & 16 & 0.0 \\
\hline 104 & PI 494751 & Uzbekistan & 18.5 & 17 & 5.0 & 14.4 & 5 & 0.0 & 33.3 & 83 & 0.0 \\
\hline 19 & NSL 6090 & USA & 46.4 & 93 & 5.0 & 14.9 & 7 & 0.0 & 13.2 & 12 & 0.0 \\
\hline 47 & PI 176371 & Italy & 27.6 & 44 & 5.0 & 15.3 & 8 & 7.0 & 16.6 & 21 & 0.9 \\
\hline 74 & PI 254565 & Afghanistan & 43.0 & 87 & 5.6 & 16.3 & 9 & 6.4 & 18.3 & 24 & 0.0 \\
\hline 33 & PI 169689 & Turkey & 37.1 & 70 & 5.0 & 17.0 & 10 & 0.8 & 28.1 & 61 & 0.0 \\
\hline 22 & NSL 6692 & USA & 56.6 & 107 & 5.0 & 18.5 & 11 & 0.0 & 19.1 & 29 & 1.5 \\
\hline 93 & PI 368825 & Yugoslavia & 19.3 & 19 & 5.0 & 18.6 & 12 & 0.0 & 32.7 & 81 & 0.0 \\
\hline 32 & PI 169688 & Turkey & 33.3 & 61 & 5.0 & 18.8 & 13 & 0.3 & 22.1 & 40 & 0.0 \\
\hline 112 & PI 604777 & Japan & 14.3 & 9 & 5.0 & 19.6 & 14 & 8.3 & 33.2 & 82 & 4.0 \\
\hline 44 & PI 175928 & Turkey & 22.6 & 34 & 5.0 & 19.6 & 15 & 0.3 & 20.1 & 33 & 0.0 \\
\hline 48 & PI 176372 & Italy & 50.1 & 100 & 5.0 & 20.0 & 17 & 1.3 & 15.3 & 17 & 0.6 \\
\hline 81 & PI 274056 & & 47.7 & 98 & 15.6 & 61.7 & 115 & 13.0 & 18.4 & 26 & 6.7 \\
\hline 63 & PI 200882 & Afghanistan & 91.1 & 120 & 5.0 & 63.6 & 116 & 20.0 & 30.3 & 72 & 0.3 \\
\hline 12 & NSL 32678 & USA & 31.1 & 53 & 41.7 & 63.6 & 117 & 5.6 & 32.5 & 80 & 0.0 \\
\hline 31 & PI 169685 & Turkey & 35.7 & 67 & 5.0 & 65.8 & 118 & 10.7 & 22.5 & 42 & 0.3 \\
\hline 39 & PI 173972 & India & 88.6 & 118 & 47.2 & 66.5 & 119 & 40.3 & 80.9 & 127 & 8.0 \\
\hline 8 & NSL 101759 & USA & 90.6 & 119 & 43.0 & 66.7 & 120 & 74.7 & 61.9 & 120 & 47.5 \\
\hline 54 & PI 179508 & Iraq & 51.8 & 104 & 5.0 & 69.1 & 121 & 57.0 & 64.8 & 123 & 13.1 \\
\hline 59 & PI 181964 & Syria & 17.8 & 14 & 36.7 & 70.0 & 122 & 0.0 & 45.3 & 108 & 0.8 \\
\hline 99 & PI 419218 & Hong Kong & 22.2 & 32 & 5.0 & 71.6 & 123 & 9.3 & 53.8 & 115 & 0.3 \\
\hline 71 & PI 229731 & Iran & 57.8 & 108 & 5.0 & 74.8 & 125 & 52.7 & 40.4 & 100 & 4.2 \\
\hline 107 & PI 508504 & S. Korea & 63.1 & 110 & 15.2 & 75.8 & 126 & 7.5 & 33.9 & 84 & 0.7 \\
\hline 65 & PI 209645 & Iran & 36.5 & 68 & 28.7 & 78.0 & 127 & 79.5 & 64.7 & 122 & 3.9 \\
\hline 103 & PI 491261 & Greece & 18.1 & 15 & 5.0 & 84.0 & 128 & 44.0 & 43.8 & 104 & 0.3 \\
\hline 120 & PI 608762 & Thailand & 67.7 & 111 & 12.2 & 92.7 & 129 & 41.5 & 89.9 & 128 & 1.7 \\
\hline 84 & PI 274311 & Pakistan & 70.4 & 112 & 33.3 & 99.3 & 130 & 75.3 & 93.8 & 130 & 24.3 \\
\hline & Tukey's ${ }^{v}$ & & 71.0 & & & 58.3 & & & 47.4 & & \\
\hline
\end{tabular}

${ }^{2}$ USDA North Central Regional Plant Introduction Station (NCRPIS) accessions are sorted by ranking of severity ratings from Trial 2 (2007). Severity of Verticillium wilt was evaluated 50 to $52 \mathrm{~d}$ after planting using the following scale: $0=$ no symptoms $(0 \%$ disease severity), $1=1 \%$ to $10 \%$ of the plant showing chlorosis and necrosis, $2=11 \%$ to $25 \%$ chlorosis and necrosis, $3=26 \%$ to $50 \%$ chlorosis and necrosis, $4=51 \%$ to $75 \%$ chlorosis and necrosis, $5=76 \%$ to $99 \%$ chlorosis and necrosis, and $6=$ plant dead. Disease scoring was based on each plant and the results converted to percentage values. The values were then averaged for all plants/pot (experimental unit). Treatments included spinach accessions (120, 130, and 130 evaluated in 2006, 2007, and 2008, respectively) and V. dahliae inoculation treatments (infested with $1 \mathrm{~g}$ colonized vermiculite $\mathrm{kg}$ potting medium vs. non-infested soil with $1 \mathrm{~g}$ non-infested vermiculite $/ \mathrm{kg}$ potting medium). The plants were incubated in a greenhouse at $25^{\circ} \mathrm{C}$ under a 12 -h photoperiod.

${ }^{y}$ Number assigned to each accession as received from the USDA-NCRPIS.

xPI 494751, Ames 23666, PI 608713, and PI 604792 are Spinacia turkestanica accessions. All other accessions are Spinacia oleracea.

${ }^{\mathrm{w}}$ LSMeans were estimated for the infested treatment in the indicated year using a randomized complete block design.

'Tukey's test for significant difference to compare entries within the infested soil treatment $(P=0.1)$ in the indicated year.

"Rank: accession ranking based on severity of Verticillium wilt.

'Because the majority of the non-inoculated entries showed no measurable variability among the replications, they were not included in the statistical analysis of the data. An arithmetric average for each of the non-infested entries is presented in the indicated year instead. 
175931, Ames 26243, PI 163309, PI 261789, and PI 494751 (Table 1). In contrast, nine accessions were rated as highly susceptible (75\% or greater), including PI 181964, PI 181808, PI 419218, PI 229731, PI 508504, PI 209645, PI 491261, PI 608762, and PI 274311 (Table 1).

ANOVA for Trial 3 (2008) also indicated statistically significant effects of spinach accession $(P<0.0001)$. The lowest disease severity ratings ( $25 \%$ or less) were observed for Ames 26243, NSL 68263, PI 175595, PI 176770, PI 204736, PI 175931, and PI 175932 (Table 1). In contrast, four accessions were rated as highly susceptible $(75 \%$ or greater), including PI 173962, PI 608762, PI 165560, and PI 274311 (Table 1).

Based on the rankings of the spinach accessions for disease severity across all three trials, four spinach accessions were consistently rated as partially resistant to Verticillium wilt (disease severity $25 \%$ or less): PI 175931, Ames 26243, PI 163309, and PI 261789 (Table 1; Fig. 1A). In contrast, 15 accessions were consistently located in the upper quartile for disease severity, i.e., highly susceptible to Verticillium wilt (Fig. 1B). When mean disease severity ratings from infested and non-infested treatments were analyzed for correlation between pairs of

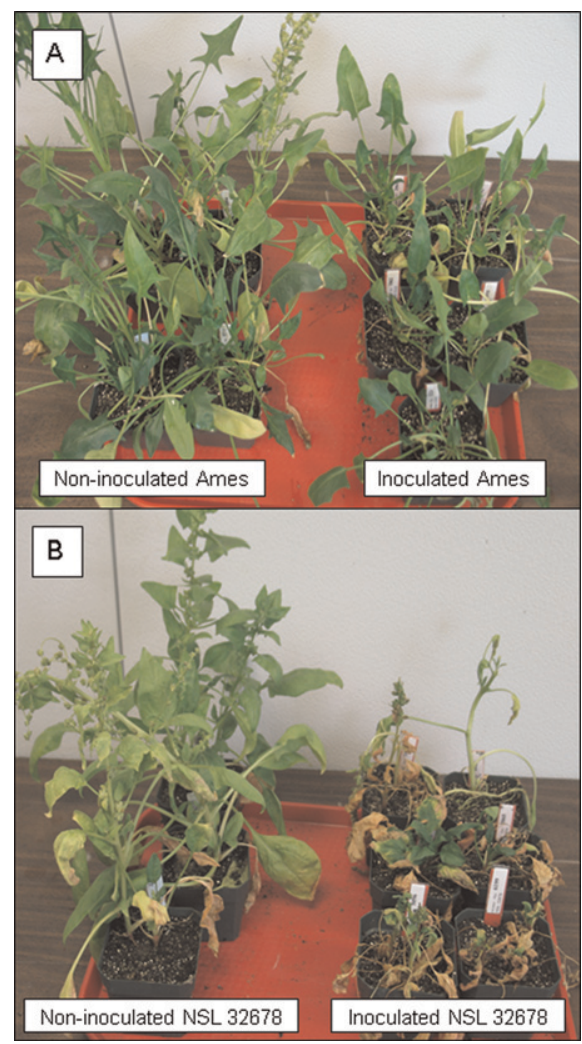

Fig. 1. USDA Spinacia oleracea accessions Ames 26243 (A), showing a resistant reaction to infection by Verticillium dahliae (mean disease severity rating $10.6 \%$ in Trial 2 , completed in 2007), and NSL 32678 (B), showing a susceptible reaction to infection by Verticillium dahliae (mean disease severity $63.6 \%$ or less in Trial 2). Plants were inoculated by sowing seed into potting medium infested with $V$. dahliae, as described in the text. years, there was a significant positive correlation in severity ratings between each pair of years: $r=0.37(P=0.0418)$ between Trials 1 and $2, r=0.35(P=0.0001)$ between Trials 1 and 3 , and $r=0.63(P=0.0001)$ between Trials 2 and 3 (Fig. 2).

In Trial 2 (2007), Lazio had the least severe Verticillium wilt symptoms of the 10 commercial spinach hybrids evaluated for resistance to Verticillium wilt (14.7\%) followed by Viroflay (19.7\%), Lion (20.9\%), Avenger (22.2\%), Polka (30.4\%), Campania (31.0\%), Califlay (34.5\%), Dolphin (41.6\%), Bolero (47.9\%), and Resistoflay (55.0\%). In Trial 3 (2008), Lazio again had the least severe Verticillium wilt of the commercial hybrids (12.9\%) followed by Polka (16.0\%), Avenger (21.0\%), Viroflay (21.4\%), Bolero (34.7\%), Campania (35.4\%), Lion (41.5\%), Califlay $(41.7 \%)$, Dolphin $(44.4 \%)$, and Resistoflay (51.3\%) (Table 2). Although male plants tended to senesce faster than female plants after initiation of bolting in the resistance screening trials, there was no apparent difference in overall disease severity ratings between male and female plants of the accessions and hybrids (data not shown).

In Trial 2 (2007), plants in infested potting mix appeared more stunted and more severely symptomatic than plants in non-infested potting mix. Thus, the impact of planting seed into infested vs. non-infested potting mix on spinach biomass was determined. Over $90 \%$ of the accessions showed a significant reduction in dry aerial biomass compared with the corresponding non-infested treatment (data not shown) with 95 of 130 accessions showing a biomass reduction of greater than $30 \%$ and 65 of the accessions showing a biomass reduction of greater than $50 \%$. The mean plant biomass for plants in infested and non-infested potting mix was 0.65 and $1.26 \mathrm{~g} /$ plant, respectively. Additionally, there were significant differences among accessions for biomass of plants grown in infested potting mix $(P<$ 0.0001). A significant positive correlation between biomass reduction and disease severity $(r=0.31 ; P=0.0078)$ was observed (Fig. 3$)$.
Conidiophores and/or microsclerotia of $V$. dahliae were observed on $100 \%$ of the petioles sampled from plants grown in infested potting medium. For accessions planted in non-infested medium, conidiophores and/or microsclerotia of $V$. dahliae were observed on $45 \%$ of the accessions (59 of 130) in Trial 2 and $7 \%$ of the accessions (nine of 130) in Trial 3. The incidences of infection among the 59 accessions varied from $11 \%$ to $100 \%$ (i.e., one to nine out of nine plants/entry from which isolations were carried out developed conidiophores and/or microsclerotia of $V$. dahliae). A majority of the infected accessions had low incidences of $V$. dahliae infection in the petioles ( $5 \%$ to $55 \%$ of the petioles/accession). However, five accessions had high incidences of $V$. dahliae infection in the petioles $(60 \%$ to $100 \%$ of the petioles/ accession).

Freeze-blotter seed health assay. The seed health assay demonstrated that $V$. dahliae was present on seeds of $34 \%$ (20 of 59) of the accessions for which subsamples of seed were assayed in Trial 2 (data not shown). In Trial 3, when seed assays were performed for all 130 accessions, $16 \%$ (21 of 130) of the USDA seed lots were infested or infected with Verticillium spp. Although a Verticillium sp. was recovered from $34 \%$ of the accessions in Trial 2, the infested or infected seed appear to have a small effect on disease severity ratings. For example, of the 20 accessions with detectable $V$. dahliae on the seed assayed, mean disease severity ratings for plants in the non-infested potting mix were low (less than 10\%) for 17 accessions and moderate (10\% to $30 \%)$ for three accessions. In Trial 3, only three accessions (PI163309, PI229731, and PI604785) of 21 on which conidiophores/microsclerotia of verticillium were observed on the seed health assay had fungal isolates identified specifically as $V$. dahliae based on ITS sequence comparisons. All three of these accessions had very low (less than $5.0 \%$ ) disease severity ratings in non-infested potting mix.

Isolates of $V$. dahliae, $V$. tricorpus, and G. nigrescens were recovered from USDA

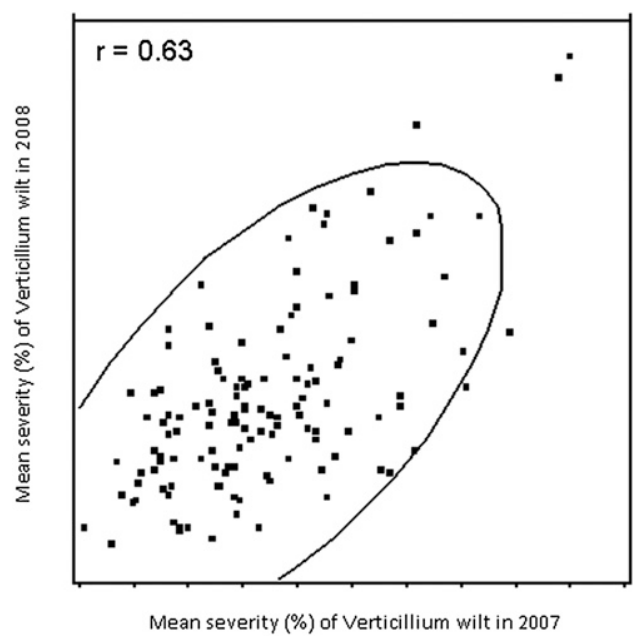

Fig. 2. Correlation and density ellipsis using a 95\% confidence interval between mean severity ratings for Verticillium wilt of 130 USDA spinach accessions screened for resistance to Verticillium dahliae in Trials 2 and 3 completed in 2007 and 2008, respectively $(r=0.63 ; P<0.0001)$. 
Table 2. Mean Verticillium wilt severity ratings (\%) for 10 commercial spinach hybrids planted into potting medium that was infested or not infested with Verticillium dahliae in each of two greenhouse trials. ${ }^{\mathrm{z}}$

\begin{tabular}{|c|c|c|c|c|c|c|}
\hline \multirow{2}{*}{$\begin{array}{l}\text { Spinach } \\
\text { entry }\end{array}$} & \multirow{2}{*}{$\begin{array}{c}\text { Commercial } \\
\text { hybrid }^{\mathrm{y}}\end{array}$} & \multirow{2}{*}{$\begin{array}{c}\text { Seed } \\
\text { company }\end{array}$} & \multicolumn{2}{|c|}{ Trial 2 (2007) } & \multicolumn{2}{|c|}{ Trial 3 (2008) } \\
\hline & & & Infested $^{\mathrm{x}}$ & Non-infested $^{v}$ & Infested $^{\mathrm{x}}$ & Non-infested $^{v}$ \\
\hline 121 & Viroflay & Chriseed & 19.7 & 0.3 & 21.4 & 1.7 \\
\hline 122 & Resistoflay & Chriseed & 55.0 & 0.0 & 51.3 & 2.4 \\
\hline 123 & Califlay & Chriseed & 34.5 & 0.6 & 41.7 & 0.3 \\
\hline 124 & Polka & Seminis & 30.4 & 0.0 & 16.0 & 0.8 \\
\hline 125 & Bolero & Seminis & 47.9 & 0.0 & 34.7 & 1.1 \\
\hline 126 & Campania & Pop Vriend & 31.0 & 0.6 & 35.4 & 2.8 \\
\hline 127 & Dolphin & Rijk Zwaan & 41.6 & 0.0 & 44.4 & 2.7 \\
\hline 128 & Avenger & Seminis & 22.2 & 5.8 & 21.0 & 0.3 \\
\hline 129 & Lion & Rijk Zwaan & 20.9 & 0.0 & 41.5 & 0.3 \\
\hline 130 & Lazio & Pop Vriend & 14.7 & 0.0 & 12.9 & 0.0 \\
\hline & Tukey's ${ }^{w}$ & & 58.3 & & 47.4 & \\
\hline
\end{tabular}

${ }^{\mathrm{z}}$ Severity of Verticillium wilt was evaluated 50 to $52 \mathrm{~d}$ after planting using the following scale: $0=$ no symptoms ( $0 \%$ disease severity), $1=1 \%$ to $10 \%$ of the plant showing chlorosis and necrosis, $2=11 \%$ to $25 \%$ chlorosis and necrosis, $3=26 \%$ to $50 \%$ chlorosis and necrosis, $4=51 \%$ to $75 \%$ chlorosis and necrosis, $5=76 \%$ to $99 \%$ chlorosis and necrosis, and $6=$ plant dead. Disease scoring was based on each plant and the results converted to percentage values. The values were then averaged for all plants/pot (experimental unit). Treatments included spinach accessions (120, 130, and 130 evaluated in 2006, 2007, and 2008, respectively) and $V$. dahliae inoculation treatments (infested with $1 \mathrm{~g}$ colonized vermiculite $/ \mathrm{kg}$ potting medium vs. non-infested soil with $1 \mathrm{~g}$ non-infested vermiculite/kg potting medium). The plants were incubated in a greenhouse at $25^{\circ} \mathrm{C}$ under a 12 -h photoperiod.

${ }^{\mathrm{y}}$ Ten commercial spinach hybrids, that serve as a set of differentials for race identification of Peronospora farinosa f. sp. spinaceae, were included in Trial 2 (2007) and Trial 3 (2008), respectively.

${ }^{x}$ LSMeans were estimated for the infested treatment in the indicated year using a randomized complete block design.

wTukey's minimum significant difference $(P=0.10)$.

vBecause the majority of the non-inoculated entries showed no measurable variability among the replications, they were not included in the formal statistical analysis of the data. An arithmetic average for each of the non-infested entries is presented for the indicated year instead.

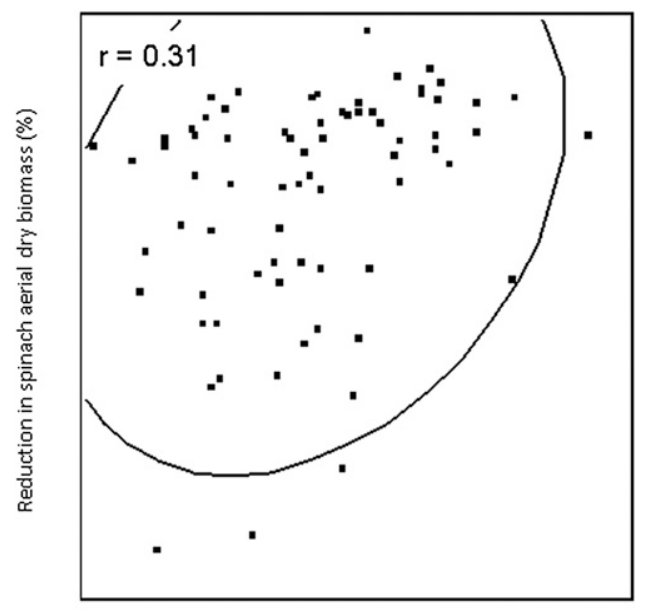

Severity $(\%)$ of Verticillium wilt in infested soil

Fig. 3. Correlation and density ellipsis using a 95\% confidence interval between mean severity ratings for Verticillium wilt and reduction in aerial biomass of plants inoculated with Verticillium dahliae compared with non-inoculated plants of 130 spinach accessions screened for resistance in $2007(r=$ $0.31 ; P=0.0078)$.

spinach accessions based on morphological examination of the cultures. Representative isolates of each of the species were subsequently examined for species identification based on the DNA sequence of the ITS region. The representative isolates of $V$. dahliae (isolate VD4), V. tricorpus (isolate JD6), and G. nigrescens (isolate LD10) (Zare et al., 2007) were confirmed as the respective species based on a BLAST match of greater than cated that no accession appeared immune to infection by $V$. dahliae, and it is unlikely that qualitative or major resistance to $V$. dahliae was present in any of the accessions tested based on the wide range in severity of symptoms observed. However, substantial differences in disease severity ratings were observed among the accessions. Low disease severity ratings were observed consistently on five entries, namely PI 175931, Ames 26243, PI 163309, PI 261789, and PI 494751. To our knowledge, no previous studies have screened for resistance to Verticillium wilt in spinach. However, Laguna (2000) screened Spinacia accessions for reactions to the soilborne pathogen Fusarium oxysporum f. sp. spinaciae. When comparing the accessions putatively identified as partially resistant to Verticillium wilt in this study with accessions identified as resistant to Fusarium wilt by Laguna (2000) in a greenhouse screening effort, there was no correlation between disease reactions to the two vascular wilt pathogens (data not shown).

In this study, evaluation of resistance to Verticillium wilt was based on visual observation of severity of disease symptoms on plants and recovery of the pathogen from infected plant (petioles). Symptoms appeared $\approx 4$ to 5 weeks after planting, when the plants started to bolt or had already bolted. These results coincided with those of du Toit et al. (2005) who reported that symptoms of Verticillium wilt on spinach were not observed until after the initiation of bolting, and Koike et al. (1994) who mentioned that symptoms on cauliflower were only observed after initiation of flowering. Approximately $50 \mathrm{~d}$ after planting, expression of symptoms of Verticillium wilt peaked, whereas natural senescence was minimal for most of the spinach accessions evaluated. By 57 to $64 \mathrm{~d}$ after planting, senescence had occurred for most accessions, when yellowing of the lowest leaves associated with natural senescence was difficult to separate from symptoms caused by $V$. dahliae. Additionally, differences were observed in severity of Verticillium wilt symptoms among plants within pots. This may have been the result of genetic variability within the accessions, the phenological stage of each plant at the time of rating, and/or variation in pathogen colonization of the inoculum substrate.

Two hypotheses might explain why $V$. dahliae was isolated from plants growing in non-infested potting medium. One is that $V$. dahliae infection occurred as a result of seed transmission from infested seed obtained from the USDA-NCRPIS. The other is that cross contamination may have occurred during the trials. Seed transmission of $V$. dahliae in spinach is now well documented (du Toit et al., 2005). To test for the possibility of infested seed serving as the source of inoculum for seed planted into non-infested potting medium, a freeze-blotter seed health assay was conducted using the limited samples of seed available for 59 of the spinach accessions. Results showed that seed samples of $34 \%$ of the accessions ( 20 of 59 assayed) were infested with Verticillium spp., i.e., 
seedborne inoculum and seed transmission were most likely involved in generating symptoms of Verticillium wilt in plants growing in non-infested potting medium. For some accessions in which plants developed symptoms of Verticillium wilt in the non-infested treatment, $V$. dahliae was not detected on the limited number of seed assayed. This suggests that either cross contamination may have occurred or the very limited number of seeds available to assay for each accession was too minimal for accurate assessment of the percentage of seed infected or infested for each accession, because infection levels as low as $0.25 \%$ have been detected on seed lots (du Toit et al., 2005).

Screening for resistance to $V$. dahliae in spinach may help develop more effective strategies to manage Verticillium wilt. Based on this study in which spinach accessions with putative partial resistance to $V$. dahliae were identified, the mechanism(s) of resistance could be in the form of reduced infection rates, reduced systemic movement of the pathogen in plants, reduced impact of infection on plant biomass, and/or reduced rate of seed infection and/or transmission. In this effort to quantify resistance to Verticillium wilt in spinach, disease severity ratings were confounded by several factors: 1) natural senescence, which occurred when the plants had flowered and set seed, mimicking the development of Verticillium wilt symptoms; 2) the spinach accessions differed in the minimum daylength required to induce bolting, which affected the timing of the onset of Verticillium wilt symptom development; 3 ) the presence of $V$. dahliae on seeds of some of the accessions highlighted the importance of including both inoculated and non-inoculated treatments in resistance evaluations to accommodate the fact that this pathogen is prevalent in commercial and Genebank seed collections; and 4) there was significant variation in severity of Verticillium wilt symptoms among plants of a given accession. Regarding disease evaluations, all genotypes were bolting by the time disease severity ratings were initiated, which was 1 week after the first genotypes to bolt were observed. Bolting had thus been initiated in most genotypes within a few days and all genotypes within a 1-week period. Thus, the disease ratings at the four rating periods were done and all disease severity ratings used in the analysis presented were 50 to $51 \mathrm{~d}$ after planting.

Regardless of the complicating variables outlined, $V$. dahliae clearly accelerated the rate of senescence of spinach plants as determined by comparing disease ratings in infested vs. non-infested potting medium. Furthermore, overall plant vigor, measured as biomass, was significantly reduced on plants growing in $V$. dahliae-infested vs. non-infested medium. This biomass reduction of infected plants is in agreement with what has been observed for other crops (Koike et al., 1994; Vallad and Subbarao, 2008; Xiao and Subbarao, 1996). A statistically significant positive correlation between reduction in biomass and severity of Verticillium wilt symptoms was observed; however, this correlation was not strong enough to predict the resistance response of any particular accession to Verticillium wilt based on biomass reduction of plants growing in infested potting medium.

\section{Conclusions}

In this study, we reported the identification of accessions with different levels of quantitative resistance to Verticillium wilt, although we did not observe evidence of qualitative resistance in the screened accessions from the USDA germplasm collection or among 10 commercial spinach hybrids tested. $V$. dahliae infections significantly reduced plant biomass, and biomass reduction was significantly positively correlated with severity of Verticillium wilt among the accessions tested. These results are valuable for characterizing potential genetic variability within spinach germplasm for the reaction to $V$. dahliae inoculation. Furthermore, these findings are critical for the identification and potential deployment of resistant sources of spinach to Verticillium wilt.

\section{Literature Cited}

Atallah, Z.K., K. Maruthachalam, L.J. du Toit, S.T. Koike, R.M. Davis, S.J. Klosterman, R.J. Hayes, and K.V. Subbarao. 2010. Population analyses of the vascular plant pathogen Verticillium dahliae detect recombination and transcontinental gene flow. Fungal Genet. Biol. $47: 416-422$.

Atibalentja, N. and D.M. Eastburn. 1998. Verticillium dahliae resistance in horseradish germplasm from the University of Illinois collection. Plant Dis. 82:176-180.

Bejarano-Alcazar, J., A.J. Termorshuizen, and R.M Jimenez-Diaz. 1999. Single-site root inoculations on eggplant with microsclerotia of Verticillium dahliae. Phytoparasitica 27: 279-289.

Brandenberger, L.P., J.C. Correll, and T.E. Morelock. 1991. Identification and cultivar reactions to a new race (race 4) of Peronospora farinosa f. sp. spinaciae on spinach in the United States. Plant Dis. 75:630-634.

Cirulli, M., F. Ciccarese, and M. Amenduni. 1994. Evaluation of Italian clones of artichoke for resistance to Verticillium dahliae. Plant Dis. $78: 680-682$

Correll, J.C., B.H. Bluhm, C. Feng, K. Lamour, L.J. du Toit, and S.T. Koike. 2011. Spinach: Better management of downy mildew and white rust through genomics. Eur. J. Plant Pathol. 129: 193-205.

Correll, J.C., T.E. Morelock, M.C. Black, S. Koike, L.P. Brandenberger, and F.J. Dainello. 1994. Economically important diseases of spinach. Plant Dis. 78:653-660.

Daehnfeldt, L. 2007. Danish production of vegetable seed-Is it only spinach? 18 June 2007. <http:// www.daehnfeldt.com/index.asp?NyhedsId $=38>$.

Diwan, N., R. Fluhr, Y. Eshed, D. Zamir, and S.D. Tanksley. 1999. Mapping of $V e$ in tomato: A gene conferring resistance to the broad-spectrum pathogen, Verticillium dahliae race 1. Theor. Appl. Genet. 98:315-319.

Domsch, K.J., W. Gams, and T.H. Anderson. 1993. Verticillium Nees ex Link 1824, p. 828-845. In:
Compendium of soil fungi. Vol. 1. IHW-Vertag, Eching, Germany.

du Toit, L.J., M.L. Derie, and P. Hernandez-Perez. 2005. Verticillium wilt in spinach seed production. Plant Dis. 89:4-11.

Foss, C.R. and L.J. Jones. 2005. Crop profile for spinach seed in Washington. U.S. Dept. Agric. National Pest Management Centers.

Grogan, R.G., N. Ioannou, R.W. Schneider, M.A. Sall, and K.A. Kimble. 1979. Verticillium wilt on resistant tomato cultivars in California: Virulence of isolates from plants and soil and relationship of inoculum density to disease incidence. Phytopathology 69:1176-1180.

Harrison, N.A. and C.H. Beckman. 1982. Time/ space relationships of colonizations and host response in wilt-resistant and wilt-susceptible cotton (Gossypium) cultivars inoculated with Verticillium dahliae and Fusarium oxysporum f. sp. vasinfectum. Physiol. Plant Pathol. 21:193-207.

Hayes, R.J., L.K. McHale, G.E. Vallad, M.J. Truco, R.W. Michelmore, S.J. Klosterman, K. Maruthachalam, and K.V. Subbarao. 2011. The inheritance of resistance to Verticillium wilt caused by race 1 isolates of Verticillium dahliae in the lettuce cultivar La Brillante. Theor. Appl. Genet. 123:509-517.

Iglesias-Garcia, A.M. 2011. Pathogenicity, virulence, and molecular characterization of Verticillium species from spinach and other hosts. MS thesis, University of Arkansas, Fayetteville, AR

Jansky, S., D.I. Rouse, and P.J. Kauth. 2004 Inheritance of resistance to Verticillium dahliae in diploid interspecific hybrids. Plant Dis. 88: 1075-1078.

Klisiewicz, J.M. 1981. Reaction of sunflower and safflower germplasm to Verticillium dahliae. Plant Dis. 65:237-239.

Koike, S.T., P. Gladders, and A.O. Paulus. 2007. Vegetable diseases. A color handbook. Academic Press, San Diego, CA.

Koike, S.T., K.V. Subbarao, R.M. Davis, T.R. Gordon, and J.C. Hubbard. 1994. Verticillium wilt of cauliflower in California. Plant Dis. 78:1116-1121.

Korolev, N. and T. Katan. 1997. Improved medium for selecting nitrate-nonutilizing (nit) mutants of Verticillium dahliae. Phytopathology 87:10671070.

Laguna, R. 2000. Screening for disease resistance to Fusarium wilt of spinach. MS thesis, University of Arkansas, Fayetteville, AR.

Metzger, J.D. and J.A.D. Zeevaart. 1985. Spinacia oleracea, p. 384-392. In: Halevy, A.H. (ed.). CRC handbook of flowering. Vol. IV. CRC Press, Boca Raton, FL.

Morelock, T.E. and J.C. Correll. 2008. Spinach breeding, p. 183-212. In: Prohens, J. and F. Nuez (eds.). Vegetables I. J Springer, New York, NY.

Mou, B., S.T. Koike, and L.J. du Toit. 2008. Screening for resistance to leaf spot diseases of spinach. HortScience 43:1706-1710.

Nicot, P. C. and D. I. Rouse. 1987. Relationship between soil inoculum density of Verticillium dahliae and systemic colonization of potato stems in commercial fields over time. Phytopathology 77:1346-1355.

Resende, M.L.V., J. Flood, J.D. Ramsden, M.G. Rowan, M.H. Beale, and R.M. Cooper. 1996. Novel phytoalexins including elemental sulphur in the resistance of cocoa (Theobroma cacao L.) to Verticillium wilt (Verticillium dahliae Kleb.). Physiol. Mol. Plant Pathol. 48:347-359.

Sackston, W.E. and F.S. Sedun. 1982. Verticillium wilt of spinach: A useful experimental system. Can. J. Plant Pathol. 4:310. 
Schaible, L., O.S. Cannon, and V. Waddoups. 1951. Inheritance of resistance to Verticillium wilt in a tomato cross. Phytopathology 41:986-990.

Simko, I., S. Costanzo, K.G. Haynes, B.J. Christ, and R.W. Jones. 2004. Linkage disequilibrium mapping of a Verticillium dahliae resistance quantitative trait locus in tetraploid potato (Solanum tuberosum) through a candidate gene approach. Theor. Appl. Genet. 108:217-224.

Smith, H.C. 1965. The morphology of Verticillium albo-atrum, $V$. dahliae, and V. tricorpus. N. Z. J. Agr. Res. 8:450-478.

Snyder, W.C. and S. Wilhelm. 1962. Seed transmission of Verticillium wilt of spinach. Phytopathology 52:365.

Vallad, G.E. and K.V. Subbarao. 2008. Colonization of resistant and susceptible lettuce cultivars by a green fluorescent protein-tagged isolate of $\mathrm{Ver}$ ticillium dahliae. Phytopathology 98:871-885.

van der Spek, J. 1972. Internal carriage of Verticillium dahliae by seeds and its consequence.
Meded. Fac. Landbouwwet. Rijksuniv. Gent. 37:567-573.

van der Spek, J. 1973. Seed transmission of Verticillium dahliae. Meded. Fac. Landbouwwet. Rijksuniv. Gent. 38:1427-1434.

Villarroel-Zeballos, M.I. 2007. Genetic, molecular, and virulence diversity of Verticillium dahliae and screening for disease resistance in spinach germplasm. MS thesis, University of Arkansas, Fayetteville, AR.

Villarroel-Zeballos, M.I., L.J. du Toit, and J.C. Correll. 2006. Genetic and molecular characterization of Verticillium dahliae from spinach and screening for disease resistance. Phytopathology 96:S118.

Villarroel-Zeballos, M.I., L.J. du Toit, and J.C. Correll. 2007. Screening for resistance to Verticillium dahliae in spinach. Phytopathology 97:S118.

Villarroel-Zeballos, M.I., L.J. du Toit, and J.C. Correll. 2008. Screening for resistance to $\mathrm{Ver}$ - ticillium dahliae in spinach. Phytopathology 98:S163-S164.

White, T.J., T. Bruns, S. Lee, and J. Taylor. 1990. Amplification and direct sequencing of fungal ribosomal RNA genes for phylogenetics, p. 315-322. In: Innes, M.A., D.H. Gelfand, J.S. Sninsky, and T.J. White (eds.). PCR protocols. Academic Press, London, UK.

Wilhelm, S. 1955. Verticillium wilt of strawberry with special references to resistance. Phytopathology 45:387-391.

Xiao, C.L. and K.V. Subbarao. 1996. Relationships between Verticillium dahliae inoculum density, wilt incidence and severity, and growth of cauliflower. Phytopathology 86:S86.

Zare, R., W. Gams, M. Starink-Willemse, and R.C. Summerbell. 2007. Gibellulopsis, a suitable genus for Verticillium nigrescens, and Musicillium, a new genus for $V$. theobromae. Nova Hedwigia 85:463-489. 